\title{
Missense mutation of TTC7A mimicking tricho-hepato-enteric (SD/THE) syndrome in a patient with very-early onset inflammatory bowel disease
}

\author{
João Farela Neves $^{\mathrm{a}, \mathrm{b}, *}$, Isabel Afonso ${ }^{\mathrm{c}}$, Luis Borrego ${ }^{\mathrm{b}, \mathrm{d}}$, Catarina Martins ${ }^{\mathrm{b}}$, Ana Isabel Cordeiro ${ }^{\mathrm{a}}$, \\ Conceição Neves $^{\mathrm{a}}$, Caroline Lacoste ${ }^{\mathrm{e}}$, Catherine Badens ${ }^{\mathrm{e}, \mathrm{f}}$, Alexandre Fabre ${ }^{\mathrm{f}, \mathrm{g}}$ \\ a Primary Immunodeficiencies Unit, Hospital Dona Estefânia, CHLC, EPE, Lisbon, Portugal \\ ${ }^{\mathrm{b}}$ CEDOC, Chronic Diseases Research Center, NOVA Medical School, Lisboa, Portugal \\ ' Gastroenterology Unit, Hospital Dona Estefânia, CHLC, EPE, Lisbon, Portugal \\ ${ }^{\mathrm{d}}$ Immunoallergy Department, Hospital CUF Descobertas, Lisbon, Portugal \\ e Laboratoire de Génétique Moléculaire, Hôpital d'enfants de la Timone, APHM, Marseille, France \\ ${ }^{\mathrm{f}}$ Faculté de Médecine, Inserm UMRS 910, Aix-Marseille Université, Marseille, France \\ ${ }^{\mathrm{g}}$ Service de Pédiatrie Multidisciplinaire, Hôpital d'enfants de la Timone, 264 rue Saint Pierre, APHM, 13005 Marseille, France
}

\section{A R T I C L E I N F O}

\section{Keywords:}

TTC7A

Very early onset-inflammatory bowel disease

TTC37

SKIVL2

\begin{abstract}
A B S T R A C T
Tricho-hepato-enteric syndrome (SD/THE) and Multiple intestinal atresia with combined immune deficiency (MIA-CID) are autosomal recessive disorders that present immunological and gastrointestinal features.

There are two different phenotypes of patients with TTC7A mutations: the severe form, caused by null mutations and leading to the classical MIA-CID; and the mild form, caused by missense mutations and leading to predominant features of VEO-IBD, less severe immunological involvement and hair abnormalities.

We expand the knowledge about TTC7A deficiency, describing a patient with the mild phenotype of TTC7A deficiency but presenting overlapping features of SD/THE and MIA-CID: intestinal atresia and inflammatory bowel disease evocative of MIA-CID, but also dental abnormalities, huge forehead, liver abnormalities, autoimmune thyroiditis and hypogammaglobulinemia, evocative of SD/THE.
\end{abstract}

\section{Introduction}

Tricho-hepato-enteric syndrome (SD/THE) is a rare autosomal recessive disorder that is classically associated with facial dysmorphism (wide forehead, hypertelorism, broad nasal root); abnormal hair (described as wooly, breakable, brittle, unbrushable and associated with trichorrhexis nodosa); chronic diarrhea and immune deficiency (mostly humoral deficiency) (Hartley et al., 2010; Fabre et al., 2011). Moreover, about half of the patients were prematurely born, present hepatic disease including cirrhosis, siderosis, hepatomegaly and have skin abnormalities like café au lait spots or xerosis (Monies et al., 2015; Fabre et al., 2013a). This syndrome is caused by mutations in the TTC37 or SKIVL2 genes, which encode for sub-units of the Ski complex, a cofactor of the cytoplasmatic exosomal RNA degradation pathway (Fabre et al., 2012, 2013b). Over the last few years, milder phenotypes have been described and even patients with isolated immune defects have been reported (Oz-Levi et al., 2015; Rider et al., 2015).

Multiple intestinal atresia with combined immune deficiency (MIA-CID) is another rare autosomal recessive disorder. It is classically associated with intestinal atresias that can occur throughout the gastrointestinal tract, particularly involving the small bowel. Short bowel syndrome due to intestinal resection and requirement of total parenteral nutrition are common features of this syndrome (Notarangelo, 2014; Chen et al., 2013). Associated combined immunodeficiency is also commonly present, allegedly caused by deficient thymopoiesis and lymphocyte differentiation, proliferation, and survival. Therefore, recurrent and opportunistic infections are also present (Notarangelo, 2014; Lemoine et al., 2014). This disease is caused by mutations in the TTC7A gene, involved in the regulation of the RhoA signaling pathway, crucial to normal ROCK function (allowing adequate cytoskeleton organization and epithelial cell polarity) (Notarangelo, 2014; Samuels et al., 2013; Fernandez et al., 2014). Recently, some patients with MIACID having only mild intestinal defects and even patients without structural defects presenting only with very-early-onset inflammatory bowel disease (VEO-IBD) have been reported (Woutsas et al., 2015; Avitzur et al., 2014).

We present the clinical report of a patient with overlapping features of both syndromes that was initially diagnosed as SD/THE. Whole

\footnotetext{
* Corresponding author. Infectious Diseases Unit, Primary Immunodeficiencies Unit, Hospital Dona Estefania, Pediatric University Hospital, Rua Jacinta Marto, 1169-045 Lisbon, Portugal.

E-mail addresses: joao.farelaneves@chlc.min-saude.pt, joao.farelaneves@gmail.com (J.F. Neves).
} 


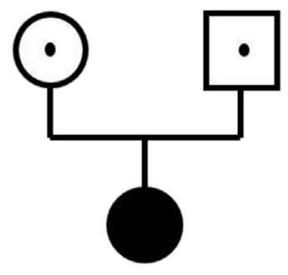

Fig. 1. Famliy predigrees showing mutation segregation. TTC7A transcript sequencing: left, variant c.1616 $\mathrm{C}>\mathrm{T}$ transmitted by the father and right, variant c.2515 G > A transmitted by the mother. The variant is highlighted in black.

\section{c.1616 C>T pSer539Leu}
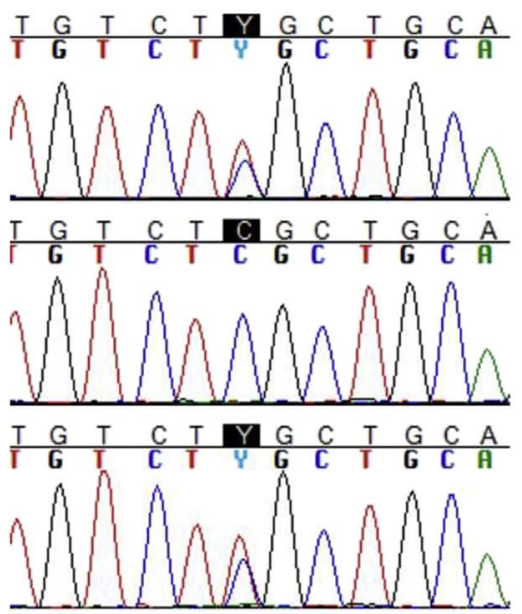

c. 2515 G>A pAla839Thr

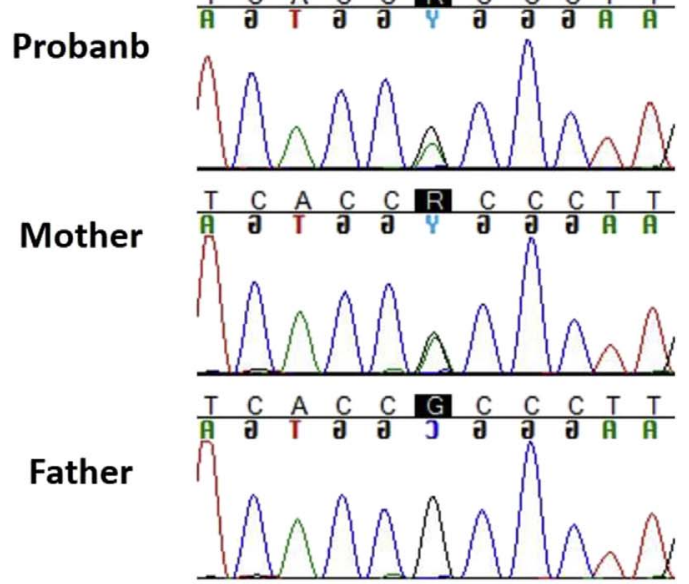

Exome Sequencing (WES) allowed the diagnosis of TTC7A deficiency.

\subsection{Clinical report}

A caucasian female infant was born at 33 weeks of gestation to unrelated parents. Her weight $(2015 \mathrm{~g})$, height $(41 \mathrm{~cm})$ and head circumference $(30 \mathrm{~cm})$ were on the 50th percentile. In the first weeks of her life, she presented feeding intolerance and at the age of two months, a type 1 ileal atresia was diagnosed. An ileostomy was performed and she was kept on both parenteral and enteral nutrition. At the age of 5 months she developed bloody diarrhea despite the presence of an ileostomy. The colonic histology revealed inflammatory pseudo-polyps, caliciform cells depletion, glandular distortion/atrophy, leading to the diagnosis of VEO-IBD. Her physical examination was notable for the presence of a prominent forehead, hypertelorism and enamel dysplasia (Fig. 2). She didn't have any hair/dermatological or cardiac

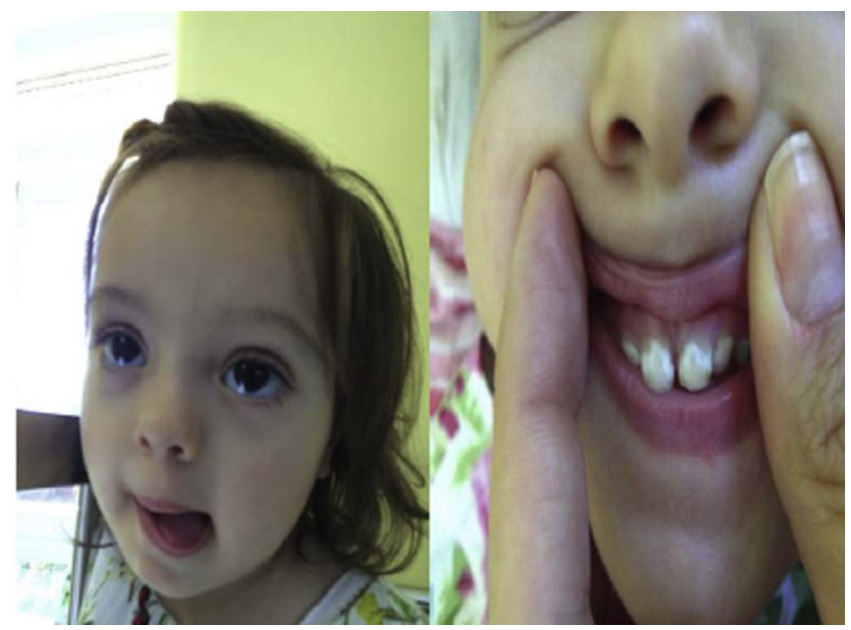

Fig. 2. Phenotype of the patient: wide forehead and hypertelorism; enamel dysplasia. abnormalities. (Relevant clinical and laboratory features are summarized in Table 1). Her colitis worsened after the re-establishment of the continuity of the intestinal tract. At the age of 20 months she started to present self-limited but recurrent elevation of AST and ALT. The hepatic biopsy revealed the presence of mild fibrosis and interface hepatitis. There were no autoantibodies related to autoimmune hepatitis present in the serum.

She also presented unexplained episodes of leukocytosis $(70-80 \%$ neutrophils) and thrombocytosis, not related to infections. The immunological evaluation showed normal lymphocyte subpopulations but severe and symptomatic hypogammaglobulinemia (recurrent respiratory infections), as well as absent serological response to vaccines. She also presented autoimmune thyroiditis.

She started subcutaneous immunoglobulin replacement at the age of 2 leading to a dramatic decrease of the number of infections. Moreover, the episodes of intermittent leukocytosis disappeared and she presented an improvement of the colonic inflammation, allowing adequate enteral nutrition.

Nevertheless, from the age of 3 to the age of 5 , she has presented 3 episodes of simultaneous elevation of AST/ALT and bloody diarrhea, which responded to low-dose prednisolone $(1 \mathrm{mg} / \mathrm{kg} /$ day $)$ and azathioprine. At the moment, at the age of 5 years-old, she has been asymptomatic for the last 12 months. She is growing on the $50^{\text {th }}$ percentile of weight, height and head circumference. Her neurodevelopment is perfectly normal.

The association of enteropathy, facial dysmorphy, dental abnormalities (enamel dysplasia), hepatic abnormalities and mild immunological involvement (isolated hypogammaglobulinemia) was evocative of SD/THE, despite the absence of hair abnormalities and the presence of a type 1 intestinal atresia (that had never been reported in $\mathrm{SD} / \mathrm{THE})$. In fact, type 1 atresia is not related to an interruption of the continuity of the intestinal tract, but is instead the presence of a membrane in the inner intestine.

However the direct sequencing of the TTC37 and SKIV2L genes revealed no mutation. A whole exome analysis was performed and showed a compound heterozygosity of TTC7A, with two missense 
Table 1

Clinical and laboratory features of the patient.

\begin{tabular}{|c|c|c|}
\hline Sex & \multicolumn{2}{|l|}{ Female } \\
\hline Current Age & \multicolumn{2}{|c|}{3 years-old } \\
\hline Gestational Age & \multicolumn{2}{|c|}{33 weeks } \\
\hline \multicolumn{3}{|l|}{ Dysmorphology } \\
\hline IUGR & \multicolumn{2}{|l|}{ No } \\
\hline Wide forehead & \multicolumn{2}{|l|}{ Yes } \\
\hline Hypertelorism & \multicolumn{2}{|l|}{ Yes } \\
\hline Skin alterations & \multicolumn{2}{|l|}{ No } \\
\hline Enamel Dysplasia & \multicolumn{2}{|l|}{ Yes } \\
\hline Hair (namely trichorrhexis nodosa) & \multicolumn{2}{|l|}{ No } \\
\hline \multicolumn{3}{|l|}{ Gastrointestinal/Nutrition } \\
\hline Intestinal atresia & \multicolumn{2}{|c|}{ Yes (Jejunal Type 1 atresia) } \\
\hline Multiple Intestinal Atresia & \multicolumn{2}{|c|}{ No } \\
\hline IBD & \multicolumn{2}{|c|}{ VEO-IBD } \\
\hline Hepatitis & \multicolumn{2}{|l|}{ Yes } \\
\hline Parenteral Nutrition (PN) & \multicolumn{2}{|c|}{$2-10$ months } \\
\hline Hematology/Immunology & Value & Reference value \\
\hline $\begin{array}{l}\text { Leukocytes }\left(\mathrm{x} 10^{3} / \mathrm{mm}^{3}\right) \text { (maximum value without } \\
\text { identifiable cause)) }\end{array}$ & 35 & $4-12$ \\
\hline $\begin{array}{l}\text { Platelets (cells/ } \mu \mathrm{L} \text { ) (maximum value without } \\
\quad \text { identifiable cause) }\end{array}$ & 950000 & $150-500.000$ \\
\hline Absolute lymphocyte count $\left(\mathrm{x} 10^{9} / \mathrm{L}\right)$ & 3600 & $2500-5500$ \\
\hline CD3 $+($ cells $/ \mu \mathrm{L})$ & 2860 & $1400-3700$ \\
\hline CD4 + (cells $/ \mu \mathrm{L})$ & 2140 & $700-2200$ \\
\hline $\mathrm{CD} 4+\mathrm{CD} 45 \mathrm{RA}+(\%)$ & $85 \%$ & $53-86 \%$ \\
\hline $\mathrm{CD} 8+($ cells $/ \mu \mathrm{L})$ & 547 & $490-1300$ \\
\hline $\mathrm{CD} 8+\mathrm{CD} 45 \mathrm{RA}+(\%)$ & $80 \%$ & $42-82 \%$ \\
\hline CD16 + /56 + (cells $/ \mu \mathrm{L})$ & 234 & $130-720$ \\
\hline CD19 + (cells $/ \mu \mathrm{L})$ & 464 & $190-1400$ \\
\hline CD19 + CD27-IgD + IgM + (\%) & $90 \%$ & $69-90 \%$ \\
\hline $\mathrm{CD} 19+\mathrm{CD} 27+(\%)$ & $8 \%$ & $7-24 \%$ \\
\hline CD19 + CD27 + IgD- (switch) & $4 \%$ & $2,7-12,5 \%$ \\
\hline CD19 + CD38 + IgMhigh (transitional) & $18 \%$ & \\
\hline $\operatorname{IgG}(g / L)$ & 3,63 & $5,3-10,7$ \\
\hline $\operatorname{IgM}(\mathrm{g} / \mathrm{L})$ & 0 & $0,46-1,9$ \\
\hline $\operatorname{IgA}(\mathrm{g} / \mathrm{L})$ & 0 & $0-1,5$ \\
\hline Anti-Diphteria & 0 & Protective $>0,5$ \\
\hline Anti-Tetanus & 0 & Protective $>0,5$ \\
\hline Free T4 (ng/dL) & 0,75 & $0,89-1,35$ \\
\hline TSH (uUI/mL) & 16,6 & $(0,7-4,1)$ \\
\hline Anti-TPO Antibodies (UI/mL) & 9,9 & $<5,5$ \\
\hline
\end{tabular}

mutations in exon 14 and 21, which were confirmed in Sanger sequencing. In fact, the patient had VEO-IBD and an intestinal atresia, which were both previously linked to TTC7A deficiency. To our knowledge, this is the first report of a patient with TTC7A deficiency who presents simultaneously an intestinal atresia and VEO-IBD and also has hepatic involvement and the phenotypic features previously described.

\subsection{Matherial and methods}

Written consent for genetic and exome analysis were obtained from the patient and her parents. The Biobank of the Department of Genetics of La Timone Hospital proceeded with the DNA extraction and ensured the long-term storage of samples. Exome sequencing was performed after exome enrichment using Agilent XT Capture Exome V5 in Illumina HiSeq (2000)/2500 platform (Aros Applied Biotechnology A/S, Denmark). The variants were first filtered with the in-house "VarAFT" system (http://varaft.eu) focusing on the 29 genes linked to congenital diarrheal syndrome to date (Table 1 SI. (Canani et al., 2015; Posovszky, 2016).

Using frequency below 0.01 on Exac Browser (http://exac. broadinstitute.org/) only one candidate gene remained: TTC7A. The patient has compound heterozygous mutations in the TTC7A gene (NG_034143.1):

- NM_001288951.1: c.2587G > A; p (Ala863Thr), inherited from the mother

- NM_001288951.1:c.1616C > T; p (Ser539Leu). Inherited from the father

Both were rare with respectively three and two alleles in Gnomad for c.2587G > A and c.1616C > T, with no description of homozygosity. Both variants were predicted to be pathogenic using bioinformatics tools (Polyphen: probably damaging; SIFT: deleterious; Mutation Taster: disease causing; UMD predictor: pathogenic). The variants were deposed in clinvar (SUB3050809). The TTC7A variants were confirmed by Sanger sequencing on a 3500XL Genetic Analyzer ${ }^{\circ}$ (ThermoFisher, Carlsbad, CA) and parental DNA samples were tested to confirm inheritance. (Fig. 1). A second analysis without gene filtering did not find any other relevant candidate gene.

\section{Discussion}

We present the case of a patient with clinical features evocative of $\mathrm{SD} / \mathrm{THE}$ but presenting missense mutations (probably hypomorphic) in TTC7A. We considered SD/THE to be the most probable diagnosis due to the presence of VEO-IBD, large forehead and the absence of combined immunodeficiency, despite the presence of a type 1 ileal atresia.

Indeed, there is significant clinical overlap of both syndromes (Table 2). This patient presents some features that had never been described before in patients with TTC7A mutations: hepatic involvement, dental anomalies, facial dysmorphism and mild immunological involvement). Additionally, even the coexistence of intestinal atresia and VEO-IBD in the same patient with TTC7A deficiency had never been described.

MIA-CID linked to TTC7A deficiency was only characterized in 2013 and in the following years a more precise characterization of the syndrome has been described. To date, there are 52 patients described worldwide and the syndrome seems to have two different phenotypes:

- Severe type, with multiple intestinal atresia and combined immunodeficiency, leading to premature death.

- Mild type, with predominant features of VEO-IBD, less severe immunological involvement (some with auto-immune features), and hair abnormalities.

Interestingly enough, in a very recent past, some patients with TTC7A deficiency were described to present some dermatological features, namely ichthyosis. This characteristic was expected to be present, since the animal model KO to TTC7A presents severe dermatological features (Leclerc-Mercier et al., 2016).

There seems to be some genotype/phenotype correlation with less severe phenotype linked to missense (probably hypomorphic) mutations, whereas the most severe phenotype is related to null mutations.

We believe that, despite the overlap that these two different conditions may present, the presence of intestinal atresia seems to be linked with TTC7A mutations, whereas woolly hair and trichorhexis nodosa

Table 2

Comparison of phenotypic feature of SD/THE, MIA-CID/VEOIBD and patients (constant: $>90 \%$ of the cases reporded, frequent: $50-70 \%$ of the cases, rare $<50 \%$ of the cases.

\begin{tabular}{llll}
\hline & SD/THE & Patient & MIA_CID/VEOIBD \\
\hline Intestinal atresia & Non recorded & Yes unique & Frequent \\
Intractable diarrhea & Constant & Yes & Frequent \\
IBD like feature & Rare & Yes & Rare \\
Facial dysmorphy & Constant & Yes & Not reported \\
Hair abnormalities & Constant & No & Rare \\
Immune deficiency & Constant & Yes & Constant \\
Liver disease & Frequent & Yes & Rare \\
IUGR & Constant & No & Not reported \\
Skin abnormalities & Frequent & No & Rare \\
\hline
\end{tabular}


are tipically linked to SD/THE. However, there are less than 60 patients with both diseases reported. Thus, a clearer vision of these syndromes will most certainly arise as more patients are described.

Finally, this case illustrates once again the importance of broad gene analysis (either targeted on a gene panel or whole exome sequencing) for the clarification of rare diseases with overlapping phenotypes.

\section{Conflict of interest statement}

The authors declare that they don't have any conflict of interests.

\section{Acknowledgements}

The exome sequencing was funded by a grant of the "Fondation maladies rares".

\section{Appendix A. Supplementary data}

Supplementary data related to this article can be found at http://dx. doi.org/10.1016/j.ejmg.2017.11.014.

\section{References}

Avitzur, Y., Guo, C., Mastropaolo, L.A., et al., 2014. Mutations in tetratricopeptide repeat domain 7A result in a severe form of very early onset inflammatory bowel disease. Gastroenterology 146 (4), 1028-1039.

Canani, R.B., Castaldo, G., Bacchetta, R., et al., 2015. Congenital diarrhoeal disorders: advances in this evolving web of inherited enteropathies. Nat. Rev. Gastroenterol. Hepatol. 12 (5), 293-302.

Chen, R., Giliani, S., Lanzi, G., et al., 2013. Whole-exome sequencing identifies tetratricopeptide repeat domain 7A (TTC7A) mutations for combined immunodeficiency with intestinal atresias. J. Allergy Clin. Immunol. 132 (3), 656-664.

Fabre, A., Martinez-Vinson, C., Roquelaure, B., et al., 2011. Novel mutations in TTC37 associated with tricho-hepato-enteric syndrome. Hum. Mutat. 32 (3), 277-281.

Fabre, A., Charroux, B., Martinez-Vinson, C., et al., 2012. SKIV2L mutations cause syndromic diarrhea, or trichohepatoenteric syndrome. Am. J. Hum. Genet. 90 (4), 689-692.

Fabre, A., Breton, A., Coste, M.E., et al., 2013a. Syndromic (phenotypic) diarrhoea of infancy/tricho-hepato-enteric syndrome. Arch. Dis. Child. 99 (1), 35-38.

Fabre, A., Martinez-Vinson, C., Goulet, O., et al., 2013b. Syndromic diarrhea/Trichohepato-enteric syndrome. Orphanet J. Rare Dis. 8, 5.

Fernandez, I., Patey, N., Marchand, et al., 2014. Multiple intestinal atresia with combined immune deficiency related to TTC7A defect is a multiorgan pathology. Medicine 93 (29), e327.

Hartley, J.L., Zachos, N.C., Dawood, B., et al., 2010. Mutations in TTC37 cause trichohepatoenteric syndrome (phenotypic diarrhea of infancy). Gastroenterology 138 (7), 2388-2398.

Leclerc-Mercier, S., Lemoine, R., Bigorgne, A.E., et al., 2016. Ichthyosis as the dermatological phenotype associated with TTC7A mutations. Br. J. Dermatol 175 (5), 1061-1064.

Lemoine, R., Pachlopnik-Schmid, J., Farin, H.F., et al., 2014. Immune deficiency-related enteropathy-lymphocytopenia-alopecia syndrome results from tetratricopeptide repeat domain 7A deficiency. J. Allergy Clin. Immunol. 134 (6), 1354-1356.

Monies, D.M., Rahbeeni, Z., Abouelhoda, M., et al., 2015. Expanding phenotypic and allelic heterogeneity of tricho-hepato-enteric syndrome. J. Pediatr. Gastroenterol. Nutr. 60 (3), 352-356.

Notarangelo, L.D., 2014. Multiple intestinal atresia with combined immune deficiency. Curr. Opin. Pediatr. 26 (6), 690-696.

Oz-Levi, D., Weiss, B., Lahad, A., et al., 2015. Exome sequencing as a differential diagnosis tool: resolving mild trichohepatoenteric syndrome. Clin. Genet. 87 (6), 602-603.

Posovszky, C., 2016. Congenital intestinal diarrhoeal diseases: a diagnostic and therapeutic challenge. Best. Pract. Res. Clin. Gastroenterol. 30 (2), 187-211.

Rider, N.L., Boisson, B., Jyonouchi, S., et al., 2015. Novel TTC37 mutations in a patient with immunodeficiency without diarrhea: extending the phenotype of trichohepatoenteric syndrome. Front. Pediatr. 3, 2.

Samuels, M.E., Majewski, J., Alirezaie, N., et al., 2013. Exome sequencing identifies mutations in the gene TTC7Ain French-Canadian cases with hereditary multiple intestinal atresia. J. Med. Genet. 50 (5), 324-329.

Woutsas, S., Aytekin, C., Salzer, E., et al., 2015. Hypomorphic mutation in TTC7A causes combined immunodeficiency with mild structural intestinal defects. Blood 125, 1674-1676. 